\title{
Influence of Network Delay and Jitter on Cooperation in Multiplayer Games
}

\author{
Anastasiia Beznosyk, Peter Quax, Karin Coninx, Wim Lamotte* \\ Hasselt University - tUL — IBBT \\ Expertise Centre for Digital Media \\ Wetenschapspark 2, B-3590 Diepenbeek (Belgium)
}

\begin{abstract}
Like most applications deployed on the Internet, modern multiplayer games are subject to the impact of transmission delays and the variability thereof. These delays can be introduced either by the physical limitations of signal transmission speed or overload and queuing problems in intermediate nodes. The influence of this delay is far-reaching and impacts most interactive applications. More specifically, quantitative and qualitative studies have been conducted on competitive game genres, such as first person shooter and racing games. In contrast, this work investigates how network delay affects player experience in cooperative games, where players have to interact with shared objects and obstacles. In this game genre, one might expect an increased sensitivity to detrimental network factors due to the reliance on the (near-)perfect synchronization of actions between participants. In this paper, a series of consecutive user tests were carried out with one of the most recent games, Little Big Planet 2; which focuses primarily on the cooperative aspect. Analysis has shown that delays over $100 \mathrm{~ms}$ significantly decrease player performance and the way in which network quality is perceived. At the same time jitter negatively affects user performance, though players do not perceive this impairment as disturbing.
\end{abstract}

Keywords: Multiplayer video games, cooperation, delay, jitter

\section{Introduction and Related Work}

In recent years there has been a substantial growth in the popularity of interactive multiplayer online games, which have become a considerable part of the Internet applications. Unfortunately, applications deployed on the Internet are often affected by network delay and jitter. In general, these factors play an important role in decreased user performance and experience. This is particularly true for multiplayer video games, given the high level of interaction between players (for various genres, e.g. sport games, first-person shooters) [Steed and Oliveira 2009; Quax et al. 2004].

Multiplayer capabilities in current games are an increasingly important revenue factor for developers, because they stimulate players to keep on playing the game after the initial release period. This leads to the users buying DLC (DownLoadable Content) and in their continued subscription to specialized networks such as Xbox Live. Typically these games focus on competitive gameplay (e.g. first person shooter games in which players are individually competing against one another).

In more recent releases and genres, cooperation between players is being utilized as the selling factor (USP). In cooperative games it

\footnotetext{
* \{anastasiia.beznosyk,peter.quax,karin.coninx,wim.lamotte\}@uhasselt.be
}

This 2011 technical report is a more elaborate version of the following publication: 10.1145/2087756.2087812. Contact author details are: anastasiia.beznosyk@uhasselt.be. This work is funded by the Special Research Fund at Hasselt University (BOF). is often impossible to succeed without help of others. One might imagine that these kinds of games are more sensitive to the network quality, as they may require very intricate and synchronized actions between several players. If one (or more) players are impaired by noticeable network delay, the gaming experience might become annoying or it can be even impossible to complete the game.

A substantial number of studies investigating the influence of network impairments (such as delay, jitter or packet loss) on the player performance in highly-interactive non-cooperative video games [Steed and Oliveira 2009] are available. Games requiring a lot of cooperation between players have not yet received much focus, mainly because their popularity is still growing. Also, the effect of the variability of delay (jitter) has received little attention, although it is an increasingly important factor in modern networks, given the universal availability of wireless connections. Both factors (cooperation and jitter) are therefore the main topics considered in this paper.

To investigate the impact of delay and jitter on cooperation, a series of consecutive user studies have been conducted. The aim is to see to what degree the player performance and experience depends on varying levels of network delay and jitter. A randomized group of players was placed in a controlled network environment. Their gaming session was impaired by introducing delay and jitter in the network connections. During these experiments, the focus was twofold: obtain objective measures (game score and task completion time) and subjective experience details (to ascertain the way in which players perceived the network quality).

A two-staged process was used. In the first experiment, a rough idea had to be gathered on what values of a more or less constant delay would lead to a decrease in experience and performance. Obviously, these figures need to be correlated to those typically found in the Internet, to avoid focusing on unrealistic test conditions. Once these boundaries are established, the second study additionally investigates the impact of jitter on the gameplay. The main goal of this study is to determine whether or not any influence of jitter (defined as variability of the delay) on cooperation in games exists and where its threshold of acceptability lies. Jitter is an important factor that is typically dependent on the last-mile technology in use [Jehaes et al. 2003] (e.g. DSL, cable or wireless connections). The goal is to investigate how this disparity between players influences the group outcome of the game.

\section{Related Work}

The influence of various network parameters (such as network delay and jitter) on user performance in multiplayer games has been widely studied [Armitage and Stewart 2004; Chen et al. 2006; Dick et al. 2005; Garapati 2009; Kim et al. 2007; Pantel and Wolf 2002; Steed and Oliveira 2009; Quax et al. 2004]. These works focus on a variety of interactive games such as first-person shooters, racing or real-time strategy games. Results have shown that users perceive the same levels of network impairment differently based on the game genre.

It is shown that games such as first-person shooter or sports games have the lowest tolerance in terms of delay, mainly because the 
player has direct control over his avatar [Dick et al. 2005]. A player constantly sees the interaction of his avatar with others as well as the environment (from a first- or third-person perspective). Therefore, one is susceptible to even low delays between the actions and the subsequent reaction of the avatar. The studies presented in [Garapati 2009; Kim et al. 2007] show that for the first-person shooter game genre, players are able to detect the presence of a (constant) delay of (at most) $100 \mathrm{~ms}$. At the same time, Quax et al. [Quax et al. 2004] show in similar fashion that for Unreal Tournament 2003 this boundary can be placed at around $60 \mathrm{~ms}$ (round trip). Pantel et al. [Pantel and Wolf 2002] analyze the impact on real-time multiplayer games using car racing simulation. They conclude that a delay up to $50 \mathrm{~ms}$ can considered non-critical for this type of game. In contrast to directly controlled games, real-time strategy games do not have such strict delay requirements [Steed and Oliveira 2009]. Here, delay may be higher without interfering with the enjoyment of the player since he just controls the units indirectly.

While being an important characteristic, jitter has not been studied so widely. In [Dick et al. 2005] authors analyze three different games (two first-person shooter games and a car racing simulator). They have shown that jitter has a negative influence on the gaming experience in general, but even with values up to $150 \mathrm{~ms}$ the environment remains acceptable. Similar results are obtained in [Armitage and Stewart 2004; Quax et al. 2004]. Here authors have shown that jitter is less significant than the delay for interactive games. A negative influence of jitter is found in [Chen et al. 2006]. Chen et al. have shown that the amount of time spent playing a game drops significantly when jitter increases.

Although previous works touch different genres of highly interactive games like racing or shooter games (which are mostly competitive in nature), the influence of network quality on cooperation in games has not been widely covered. There are several works in existence regarding the effect of network characteristics on cooperation in shared virtual environments [Park and Kenyon 1999; Stuckel and Gutwin 2008]. They have shown a significant influence of network delay and jitter on the user performance, even though the focus of these studies was clearly outside the gaming context. To our knowledge, the impact of network conditions on games that focus on cooperation has not yet been studied. Due to their nature, one might expect that cooperative games are even more sensitive to network impairments (both delay and jitter).

\section{Measurement Setup and Procedure}

As stated in the introduction, Little Big Planet (LBP) $2{ }^{1}$ was used as test subject for these experiments, as it is a current title that successfully makes use of community features and collaboration between players. In fact, levels in LBP are constructed in such a way that close cooperation is a requirement to complete them successfully.

A two-step approach is used to quantify the impact of delay and jitter. After determining the rough boundaries for the delay values (constant) in a first stage, they were refined for use in subsequent scenarios. Also, the second stage introduced jitter in order to see whether any influence exists (part 1) and, if so, what level is considered to be acceptable by players (part2).

\subsection{Study 1: Influence of Network Delay}

Thirty two participants were recruited for our first test. Although they were frequent players of multiplayer video games, most of

\footnotetext{
${ }^{1}$ http://www.littlebigplanet.com/en/2/
}

them had never played Little Big Planet 2 before. All participants were randomly grouped in pairs and played the game using two separate Sony PlayStation (PS) 3 consoles that were connected to each other over a dedicated local area network (figure 1). An uplink was provided to connect both to the PlayStation Network (required for matchmaking purposes). As the traffic associated with the game is sent directly between the consoles, the presence of a single impairment node (a linux system running the NetEM [Hemminger 2005] software) suffices to introduce network anomalies for this setup. The software is installed to run in bridge mode in order to obtain optimal performance and to reduce any possible impact from routing issues. Care was taken to choose delay and jitter values representative for current-generation network conditions (i.e. excessively high values were not considered). To ease observations, the participants were located in the same room separated by a portable wall so that they were not able to see their partner or their partner's monitor.

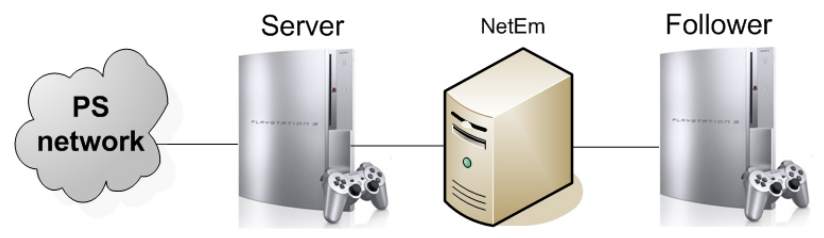

Figure 1: Network layout in the first study.

To measure the effect of delay, different network conditions were simulated. These conditions varied from a slightly delayed environment of $10 \mathrm{~ms}$ to a worst-case maximum of $300 \mathrm{~ms}$ (all numbers stated as one-way). There were 8 different conditions of 10, 20, 40, $60,80,100,200$ and $300 \mathrm{~ms}$. Of these, values between 20 and 100 $\mathrm{ms}$ can be considered typical realistic values for Internet applications [Steed and Oliveira 2009]. A delay of $10 \mathrm{~ms}$ was considered as an unimpaired environment, as players are typically not located on the same local area network. For every pair two out of eight values of delay were randomly chosen. Players were not aware what value of network delay they experienced to avoid any influence on their further responses.

For our test we selected two cooperative levels which were played at least by several members of the LBP community ${ }^{2}$ and had several positive reviews (Coop World by Lenicolas59 and COOP by I-Lex ${ }^{3}$ ). They had approximately the same difficulty level and duration. During the experiment, participants often encountered situations that required synchronizing their actions. Examples of cooperative tasks are shown in figure 2. Here, figure 2a shows a player below that has to jump between platforms which appear only if the player above pulls the appropriate trigger. Figure $2 \mathrm{~b}$ shows another example of tight cooperation where one player (held by his partner) has to jump at the moment he is being thrown by the other. Other examples of shared tasks were throwing and catching objects between two players, carrying each other while shooting, and lifting and moving the same objects.

In every pair, one of the players was impaired by the network delay. By impairment of the player we mean that his PlayStation 3 was connected through the local area network by means of the impairment node (with NetEm installed). To initiate a gaming session in Little Big Planet 2, it is necessary for one player to invite the other one. The PlayStation which sends the invitation is called game leader and acts as 'server'. The other player connects to this server and is referred to as the follower. We should point out at this

\footnotetext{
${ }^{2}$ http://lbp.me/

${ }^{3}$ Here we provide the level name and the creator's nickname
} 


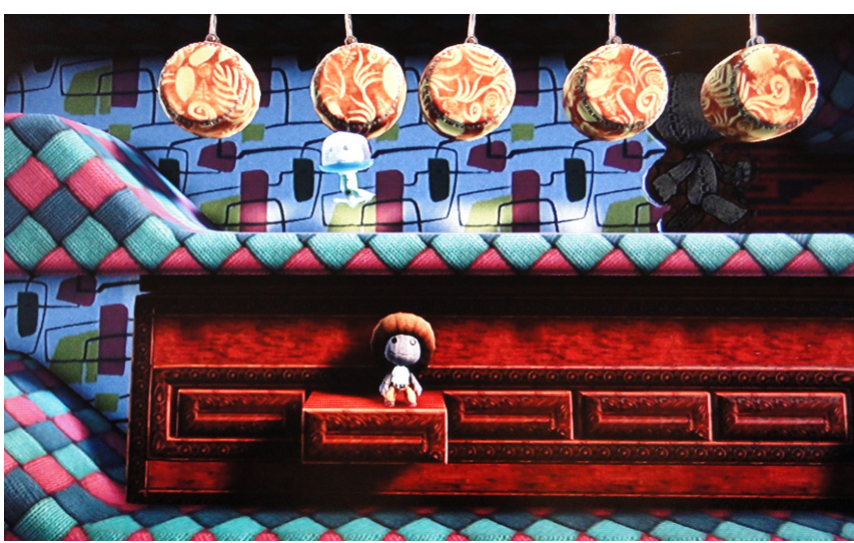

(a)

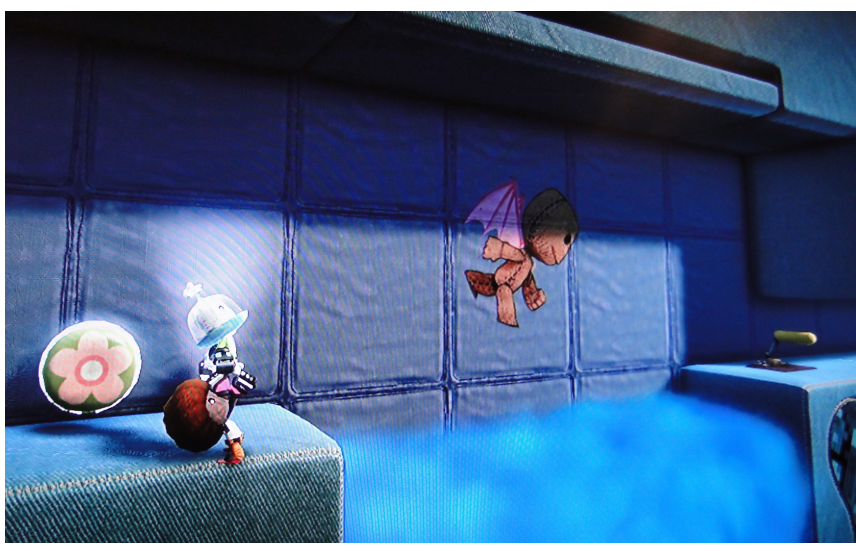

(b)

Figure 2: Examples of cooperative tasks used in the first study.

time that the exact workings of LBP2 on the network level are unknown, as the protocol has not been reverse engineered. However, we were able to deduce some of its workings based on packet traces and visual observations.

Firstly, the fact that a single party is designated as server does not mean that all network traffic is routed through this node. Actually, in a session with more than two players, data is exchanged between all parties involved (in a mesh).

Secondly, the server (being the initiating party) does play an important role in the synchronization process. This machine seems to have the final say in the synchronization of actions between the various players, as it determines the time at which actions are undertaken for the global game state. Therefore, the player that uses the server as his console typically experiences the smoothest game play, as actions are directly interpreted. For the other consoles connected to the server, a delay estimation method is used to try and determine at what time the actions would arrive at the server. They are subsequently locally delayed before being visualized. At high delay levels, this results in a short (but sometimes noticeable) delay in the execution of command on a follower console.

Third, once the server determines the current state of the game, it distributes the state among all parties involved and each of them corrects to the 'right' state. Visually, this is represented by the avatar taking steps back (in the optimal case) or moving through solid objects (worse case). Note that this technique relies heavily on the delay estimation method being used, which will probably not

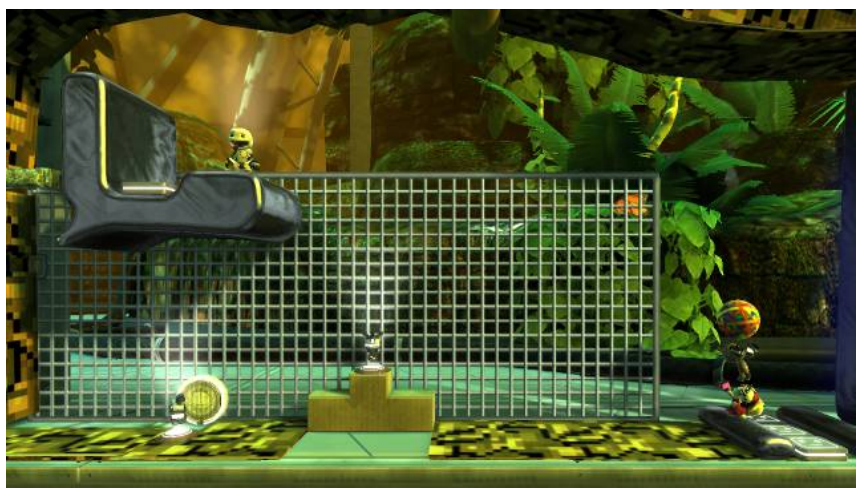

Figure 3: Level used in the second study.

yield correct results in face of jitter.

As indicated, the player using the server console is at an advantage (because actions are directly undertaken) and is therefore referred to as 'unimpaired', while the others are 'impaired'. It is important to state that the unimpaired player also experiences detrimental performance due to the fact that the actions of the other players take a while to arrive at the server (and may be out of order due to jitter), but not in the order of magnitude of the impaired players.

During the test, level completion time and game score were measured. After completion of each level, the participants were asked to fill in a questionnaire enabling them to evaluate the influence of the network conditions on their gaming experience. The questionnaire consisted of the following questions:

- Rate the quality of the network (from 1 - unacceptable environment to 5 - perfect environment)

- Rate the influence of the network quality on the following aspects of your gaming experience: enjoyment, frustration, score and completion time (from 1 - not at all to 10 - very much)

- Rate the influence of the network quality on difficulty to coordinate cooperative activities with the partner (from $1-$ not at all to 10 - very much)

- Rate the influence of the network quality on the wish to continue the game (from 1 - not at all to 10 - very much)

It took between 20 and 40 minutes for each pair to complete the test.

\subsection{Study 2: Influence of Jitter}

For both parts of our second study we involved groups of three people playing a custom level of Little Big Planet 2. Three players had to coordinate their actions in order to throw a ball into a basket. The game continued until 800 points were collected. For each successful attempt, the players scored 200 points. Figure 3 presents a part of the environment. Two players (on the right) had to throw the ball together to the third player standing on the platform (on the left). The latter had to catch the ball and throw it into the designated area.

Three PS3 consoles were connected through a switch over the LAN. In order to simulate different network conditions for all players, two impairment nodes with NetEm were placed between the central switch and the consoles as shown in figure 4. To bring the setup closer to the real playing conditions and to avoid interactions through vocal communication, every participant was located in a 


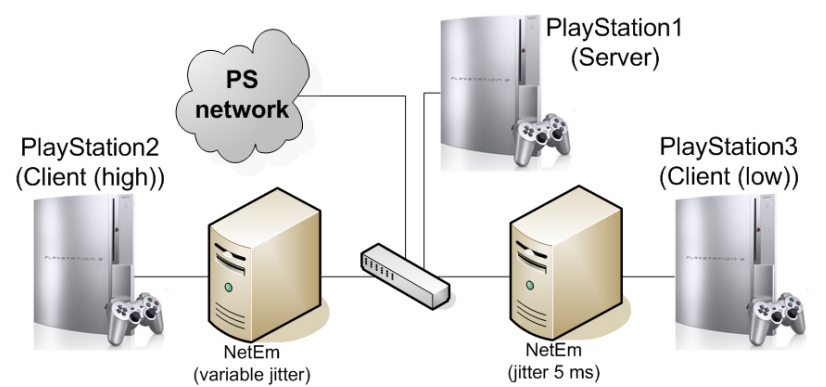

Figure 4: Network layout in the second study.

different room. The amount of jitter is represented in NetEM as a value that is superposed on the 'fixed' delay (more precisely, the figure indicates the standard deviation). In practice and as an indication, a fixed delay of $100 \mathrm{~ms}$ with a jitter of $20 \mathrm{~ms}$ will result in values that are roughly spread between $140 \mathrm{~ms}$ and $60 \mathrm{~ms}$, with the majority around $100 \mathrm{~ms}$ (due to the normal distribution chosen, as it provides more life-like results). Note that for the following discussion, values are provided for the one-way delay.

In order to analyze different amounts of jitter on user performance, the first part of the study had the following design. We fixed the amount of delay for each group and varied jitter to see whether it had any influence on players' performance. There were four groups participating in this test: the first two were exposed to $100 \mathrm{~ms}$ delay, and the other two to $200 \mathrm{~ms}$ delay. We varied the jitter values between PlayStation1 and PlayStation2 by assigning it to $20 \%$ and $50 \%$ of the fixed delay (simulating a cable access connection). These values were given in a different order to every group. At the same time, the jitter of the connection between PlayStation 1 and PlayStation 3 was fixed to $5 \mathrm{~ms}$ (which is typical for DSL connections). Every time the level was completed participants switched between the consoles. In such a way every player tried both unimpaired (PlayStation1) and impaired (PlayStation2 or PlayStation3) environments.

The second part of this study was designed similarly to the previous one and an identical setup was used (figure 4). We used the same custom level, but participants were asked to complete it as fast as possible. Twenty four people were recruited and randomly put together into eight groups. This time, a single fixed delay value was used (100 ms) and four levels of jitter were introduced: 10, 20, 40 and $50 \mathrm{~ms}$. Each group of participants tested all four levels of jitter, whose order was randomized. While testing the first given jitter value, participants switched PlayStations each time the level was completed (as earlier). To avoid too many tests for each group, for the other three jitter values players remained at the same location.

In both tests we captured the completion time. After completion of each level, the participants were asked to fill in a questionnaire similar to the one in the first study. Each group spent between 40 and 60 minutes to complete the test.

\section{Results}

This section presents the results of our studies on how network delay and jitter influence different aspects of player experience in cooperative games. For every study, we provide an objective evaluation based on the game completion time and the game score. Afterwards, we focus on responses collected through the questionnaire.

The graphs in this section represent the values obtained by averaging certain characteristic (e.g. time, score, rating) among sessions where players experience the same level of delay or jitter.

\subsection{Study 1: Influence of network delay}

First of all we analyze how a fixed network delay affects the completion time and game score. By doing this we determine objectively whether or not the network delay influences player performance. Completion time analysis has shown that with a delay higher than $100 \mathrm{~ms}$ the game lasted noticeably longer (figure 5a). As can be seen from the figure, the time to finish the game increases constantly when the delay exceeds $100 \mathrm{~ms}$. This has been also confirmed by a significant positive correlation between completion time and the level of delay $\left(R^{2}=0.82, p=0.013\right)$. Note that this value (which represents a round trip of $200 \mathrm{~ms}$ ) is becoming less and less common for players located on the same continent.

Further analysis has shown the correlation between the delay and the game score $\left(R^{2}=-0.78, p=0.024\right)$. As shown on the figure $5 \mathrm{~b}$ there is a noticeable drop in the game score once the network delay exceeds $60 \mathrm{~ms}$.

Furthermore, we find it interesting to see whether or not players have perceived this degradation. In the questionnaire, participants were asked to rate the influence of the network delay on their score and task completion time. Figure $5 \mathrm{c}$ represents the user selfreported perception of the delay impact. Players seemingly do not perceive a delay up to $200 \mathrm{~ms}$ as disturbing (a low impact on the experience level). A positive correlation has been found between delay and both completion time $\left(R^{2}=0.81, p=0.016\right)$ and game score $\left(R^{2}=0.85, p=0.008\right)$.

For each delay level players were asked to rate the gaming environment to define when it became annoying and/or unacceptable. Additionally, we wanted to know at what level they would prefer to quit the game. Results show that up to $200 \mathrm{~ms}$ (one way delay), players considered the gaming environment to be acceptable without major impairment. Only when the delay exceeded $200 \mathrm{~ms}$ did they indicate this to be very annoying. At the same time delays higher than $100 \mathrm{~ms}$ increased the likeliness of players to quit the game.

The analysis of the aforementioned characteristics has been performed based on the data collected from the players who were directly influenced by the network conditions. However, cooperative games involve simultaneous interaction between several players, both those affected and those that are not. Therefore, players who are not directly influenced by the network delay can also be affected by an inadequate performance of their game partners. In particular, not only does an impaired player experience a delay in the events on his own site, but his actions are also delayed when delivered to the remote player (as explained in section 3.1). To investigate whether an impaired player impacted his/her collaborator we have asked both of them to evaluate their gaming experience. First, players evaluated how difficult it was to coordinate shared activities, and then the level of their enjoyment and frustration (figure 6). By comparing these responses we aim to define a threshold that provides all players with an enjoyable gaming experience.

Figure 6a shows that with an increase in network delay, impaired players tend to have more difficulties coordinating actions with their partners. For unimpaired players, delays below $100 \mathrm{~ms}$ do not noticeably increase the difficulty level, which remains at a low value (below 5). On the contrary, a delay higher than 100 ms shows a constant growth of the perceived difficulty level. In both cases there is a significant correlation between delay and the difficulty level $\left(R^{2}\right.$ $=0.83, p=0.011$ ). To define the threshold of acceptable delay, values should be chosen that keep the difficulty level for both players at a relatively low level (in the lower part of the rating scale, i.c. below 5). Based on the coordination difficulty scores, there is an indication that a one-way delay below $60 \mathrm{~ms}$ does not significantly 


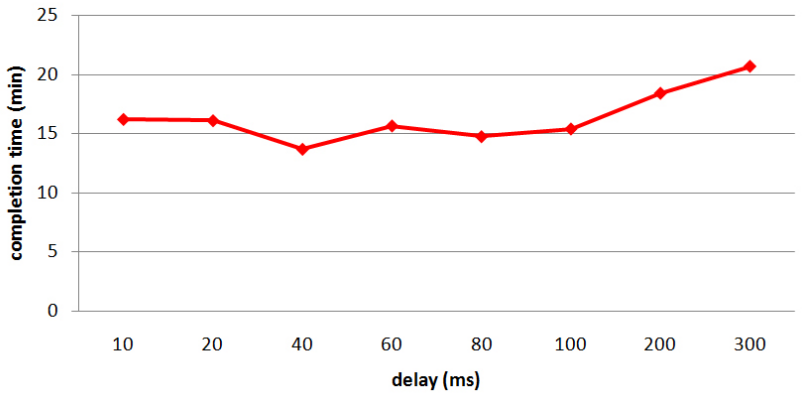

(a) Overall game completion time (objective).

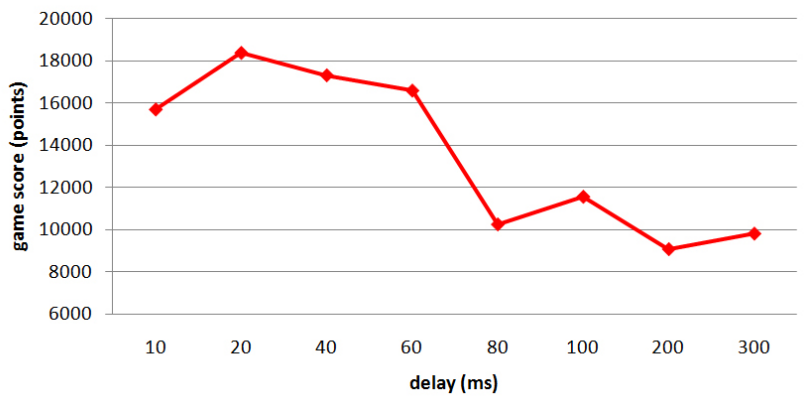

(b) Overall game score (objective).

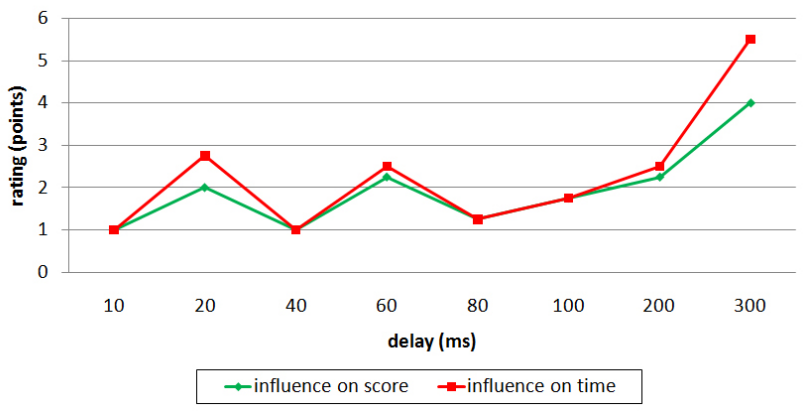

(c) Perception of network delay given by the impaired players (subjective).

Figure 5: Objective and subjective influence of network delay on the completion time and the game score.

decrease the user experience for both parties involved.

An interesting result is observed when comparing the level of enjoyment between impaired and unimpaired players (figure 6b). In those cases where delay is below $80 \mathrm{~ms}$, the unimpaired users have indicated their enjoyment slightly lower than those who were directly affected by delay. At the same time, when delay reaches $100 \mathrm{~ms}$ (and higher) enjoyment of impaired participants was considerably influenced by it. The rating of enjoyment given by the affected players has a negative trend, indicating that there is reduced enjoyment when faced with higher delays $\left(R^{2}=-0.88, p=\right.$ $0.004)$. However, ratings given by unimpaired players do not have such a strongly pronounced regularity, being more balanced around the average value $(M=8.72, S D=0.88)$. Therefore, the threshold of acceptable delay is defined here based on the evaluations by the class of impaired players. With delays higher than $100 \mathrm{~ms}$, there are indications that user enjoyment decreases constantly.

Figure $6 \mathrm{c}$ shows the difference in the level of frustration between two groups of players. There is a significant correlation between de-

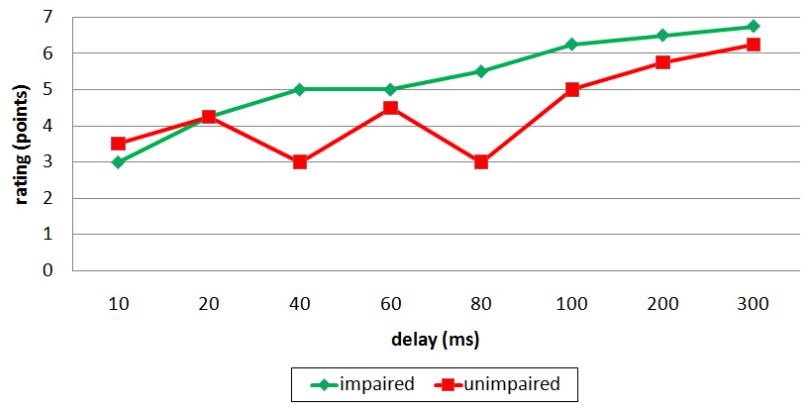

(a) Difficulty to coordinate actions.

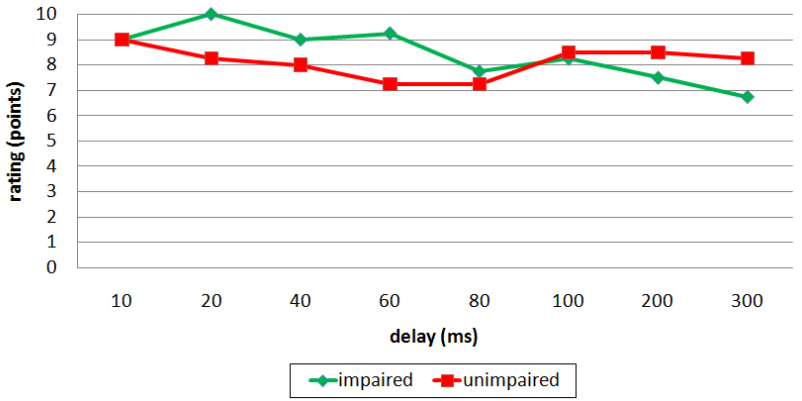

(b) User enjoyment.

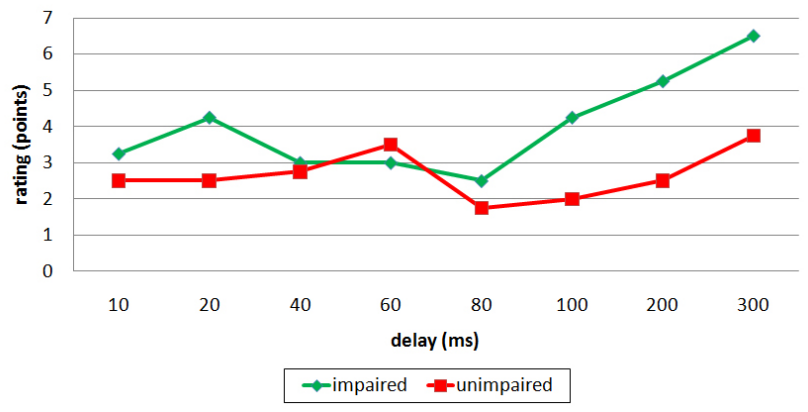

(c) Level of user frustration.

Figure 6: Influence of the delay on the impaired and unimpaired players.

lay and frustration for the impaired players $\left(R^{2}=0.85, p=0.008\right)$. We observe that after a delay of $100 \mathrm{~ms}$ the level of frustration of impaired players is increased dramatically. At the same time for non-affected players the level of frustration remains quite low (not exceeding 4) with a slight increase when the maximal delay (300 $\mathrm{ms}$ ) is reached. Here, we define the delay of $100 \mathrm{~ms}$ as a threshold below which both of the players experience low level of frustration (not exceeding 5).

Taking into account these findings delays between 60 and $100 \mathrm{~ms}$ (and below) are considered as those that provide the most enjoyable experience and adequate performance in the cooperative game being used. $100 \mathrm{~ms}$ delay is defined as a threshold above which players perceive network degradation as disturbing with a significant decrease in their performance. 


\subsection{Study 2: Influence of jitter}

Our second study investigates the influence of jitter on player experience and performance in cooperative games. It consists of two consecutive parts. First we determine whether there is any influence of jitter on cooperation (to justify further study). To accomplish this, the performance and experience of players under conditions of low and high levels of jitter is compared. To quantify the impact of jitter on cooperation, in the second part several levels of jitter are introduced. We aim to define the threshold of jitter below which players do not feel hampered in the game, when faced with the goal to finish the level as quickly as possible. Also, the discrepancy within the group of players using various network access technologies (e.g. DSL, cable connection, etc.) is studied.

\subsubsection{Part 1: Existence of jitter impact}

During this first study we want to see whether jitter has any negative influence on the cooperative play. In order to perform this we have opted for certain delay values and varied jitter between PlayStations. We considered two values of fixed delay: $100 \mathrm{~ms}$ and $200 \mathrm{~ms}$. While the first value was selected as a threshold of the game acceptability (based on the first user study), the second one was used to define the worst possible condition (keeping in mind the choice of realistic values for current generation networks).

As in the previous study, we have evaluated the impact of the network quality both objectively and subjectively. As an objective characteristic of the gaming experience we have analyzed the level completion time. We have observed that players who were exposed to lower jitter values completed the level quicker (see figure 7). Obtained results have also confirmed the finding from the first test proving that the level completion time increases with higher delays.

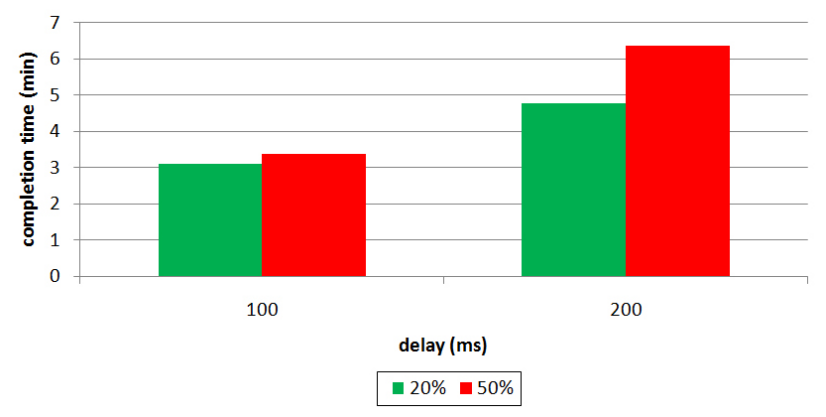

Figure 7: Influence of jitter on the completion time.

Although we have found a negative effect of jitter on player performance (objective measurement), it is still necessary to analyze whether players consider it degrading their experience (subjective). In order to reveal whether or not players feel hampered by jitter we have examined their responses given in the questionnaire.

Participants played the game in groups of three, where everyone was exposed to a different condition. One player did not experience any direct influence of jitter, while the other two were affected by it. One of the affected participants experienced varying high levels of jitter, while the other one was exposed to a constant low level of $5 \mathrm{~ms}$ jitter. We will refer to them as server, client (high) and client (low) respectively (figure 4). We reiterate the fact that NetEM uses a normal distribution to determine the delay values; the values in the following paragraphs therefore indicate the standard deviation.

All three players found the conditions acceptable without noticeable impairment for any level of jitter when delay was fixed to 100

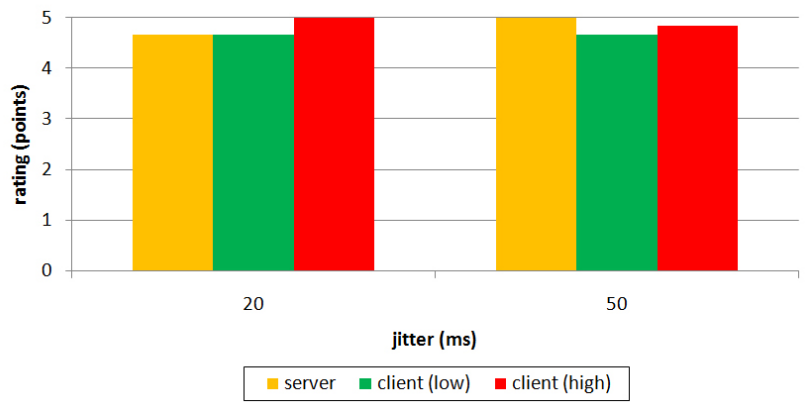

(a) 100 ms delay.

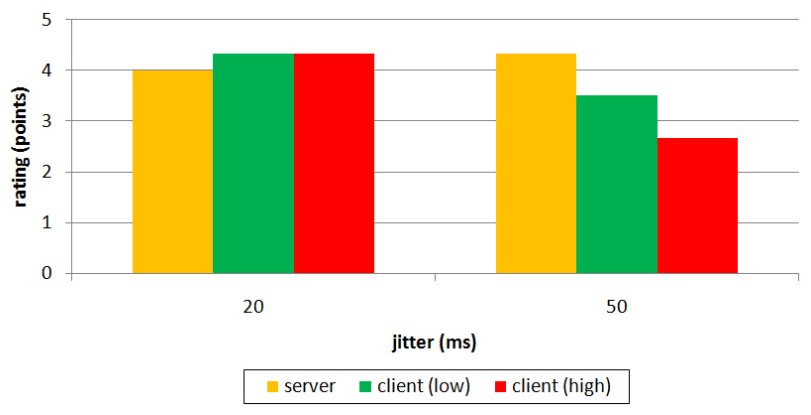

(b) 200 ms delay

Figure 8: Players' perception of the network quality.

$\mathrm{ms}$ (figure 8a). When players were subjected to $200 \mathrm{~ms}$ delay different levels of jitter were not perceived equally (figure $8 \mathrm{~b}$ ). Jitter up to $20 \%$ (or $40 \mathrm{~ms}$ ) was considered as an acceptable environment with minor impairments. Yet, high level of jitter (50\% or $100 \mathrm{~ms}$ ) significantly decreases the perceived quality of the network. The server player has not experienced negative influence, but both other players have indicated these conditions to be very annoying with many noticeable impairments. Note that this is probably due to the fact that the latency compensation techniques in LBP2 cannot efficiently cope with the variations in delay.

Further, we have analyzed users' responses regarding the influence of the network quality on their enjoyment, frustration, difficulty to coordinate joint activities, game completion time and wish to continue the game. When the delay was $100 \mathrm{~ms}$ players did not feel hampered by any of the jitter values $(5,20,50 \mathrm{~ms})$. They have indicated very low influence of the network quality and the will to continue playing under given conditions.

An opposite situation is observed for those players that are subjected to $200 \mathrm{~ms}$ delay. Firstly, there is a greater discrepancy in players' perception within the same group (between server player, client (high) and client (low)). Secondly, the analysis of results have shown the difference between responses of players that are subjected to $20 \%$ and $50 \%$ jitter, which do not occur for the $100 \mathrm{~ms}$ delay case. $20 \%$ jitter (or $40 \mathrm{~ms}$ ) is found to be the worst condition for client (high) and server player, while client (low) has indicated its influence to be less crucial. This is reflected in the players' wish to continue the game, which is lower for client (high) and server player than for client (low). During the trials with 50\% jitter (or $100 \mathrm{~ms}$ ), the server player has indicated the lowest influence of the network conditions on the gameplay among three people. For all aspects of the interaction investigated in this study, client (high) has indicated the highest influence of the network quality on his gaming experience. Client (low) has evaluated the influence of the network 
quality to be relatively high but not very different from the server player.

\subsubsection{Part 2: Quantification of jitter impact}

In the previous part we have shown that jitter negatively affects user performance in the cooperative game that is being used. We have observed that time required to complete the level increases with the level of jitter. Because we have checked this only for two different levels of jitter it is still necessary to confirm the findings with gradually increasing jitter. Therefore, we have organized a larger user study where various levels of jitter were introduced.

This time we have restricted delay to $100 \mathrm{~ms}$ only, as an acceptable threshold value. Although we have found no influence of this network condition on players' experience in earlier test (subjective), we have observed a negative impact on completion time of the cooperative game (objective). Therefore, we assume that the same network quality will be perceived differently if the goal of the task is time dependent. In order to check this assumption, the game level used in the previous test has been slightly modified. This time it was necessary not only to score a certain amount of points but also to achieve this goal as fast as possible.

First we have analyzed the influence of different levels of jitter on the task completion time. A positive significant correlation $\left(R^{2}=\right.$ $0.44, p=0.002$ ) has been found between jitter and the completion time (figure 9).

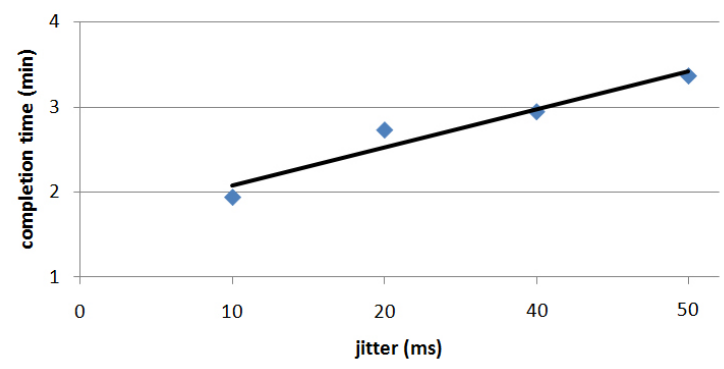

Figure 9: Influence of jitter on the completion time.

Furthermore, we have analyzed how different players perceived this degradation subjectively. Seeing the influence on the completion time, we asked players to evaluate whether or not they perceived any influence of the given network condition on the time to accomplish the task. Three players have evaluated the jitter impact differently. From the graph (see figure 10) we conclude that all three players indicated jitter impact to be very low. The server player has not perceived an increase of jitter as a degradation of his experience. His evaluation remained at the same level across the conditions. At the same time we can see an increase of jitter influence on client (high). Client (low) felt more affected than the server player, but in reality remained at the same level with exception of the highest level of jitter. While the jitter remained under $50 \mathrm{~ms}$ we have not observed a major difference between players exposed to different conditions. Only when the jitter reached $50 \mathrm{~ms}$ was the difference between impaired players and the server player elevated and greater than 1.

We have asked players to rate the overall quality of the network condition for each level of jitter. Figure 11 presents user evaluation according to the mean opinion score (MOS) gradation. As we can see jitter does not substantially decrease player perception of the network quality for the server player and client (low). At the same time client (high) experiences a gradual decrease of network

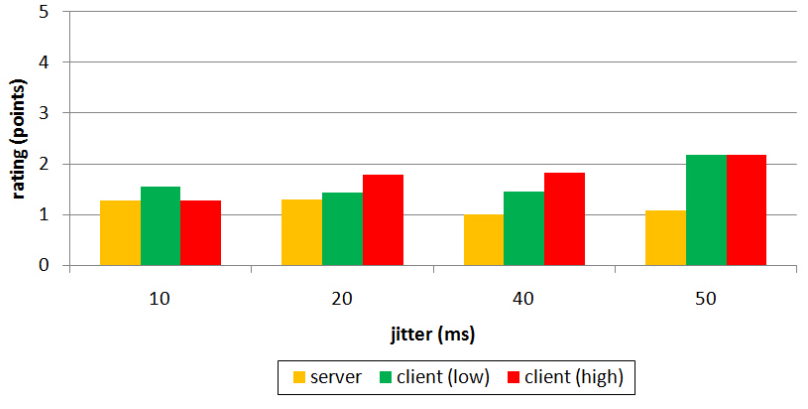

Figure 10: Influence of jitter on the completion time.

quality, which drops to a relative low when jitter reaches $50 \mathrm{~ms}$. Moreover, in cases of very high jitter the difference between player perception is more noticeable. For $50 \mathrm{~ms}$ jitter we observe that interaction between players becomes more unequal as the players experience different levels of degradation.

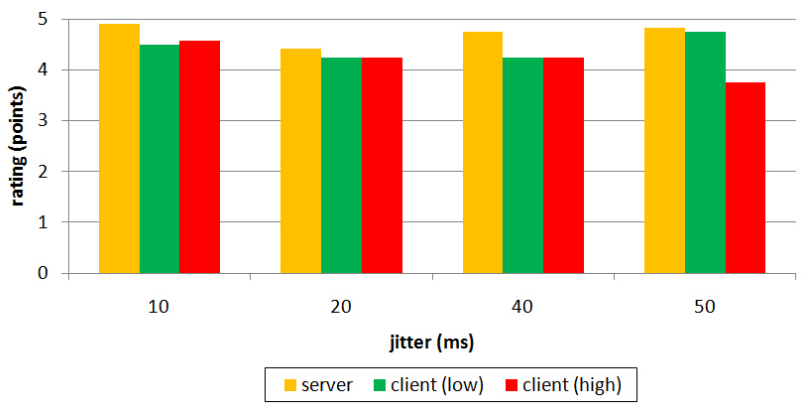

Figure 11: Influence of jitter on the network quality.

Other data gathered through the questionnaire reflects an influence of jitter on the ability to efficiently coordinate joint actions (figure 12a), player enjoyment (figure 12b) and frustration (figure 12c).

These figures show that the server player's experience has not been affected by the jitter increase. Ratings given by client (high) are somewhat different, indicating a low yet growing negative influence of jitter. For client (low) we observe a relatively similar evaluation among four conditions, with the exception of the highest level of jitter. Again we observe a greater difference between the server player and players that experience jitter. The existence of this difference between the server player and affected players confirms our assumption that there is a clear negative influence of jitter, as it unbalances the gaming experience between players.

The obtained findings have proved that higher levels of jitter have a negative influence on player experience in cooperative games (i.c. LBP2). Besides a negative impact on the performance, it also results in an unbalanced experience between players. While the discrepancy between players (caused by different access technologies) remains relatively small with low jitter level, it grows significantly when jitter reaches $50 \mathrm{~ms}$.

\section{Discussion and Conclusion}

Our studies have shown that cooperative games (i.c. Little Big Planet 2) which actively require interaction with other players are sensitive to the network quality, in particular to network delay and jitter. In our first study we analyzed the impact of delay on different factors of the gameplay, such as enjoyment, frustration, difficulty of 


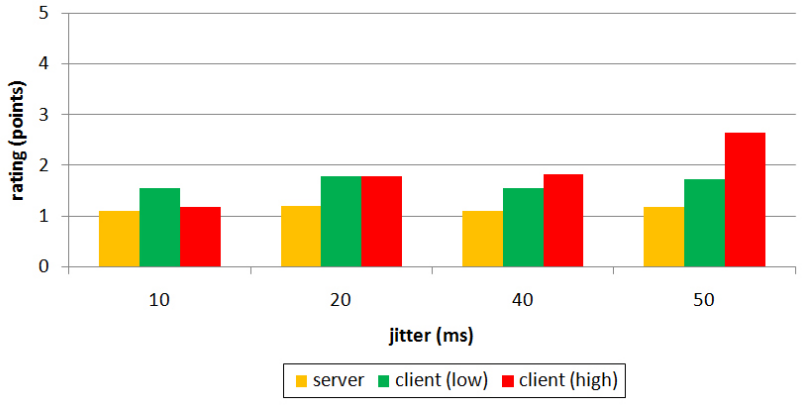

(a) Influence of jitter on the difficulty of coordination.

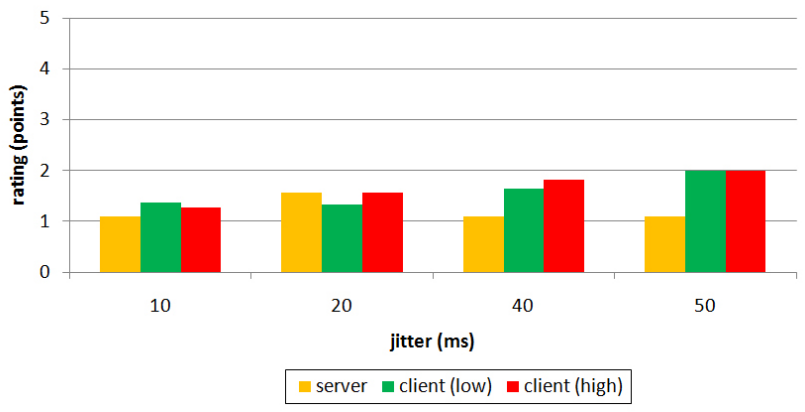

(b) Influence of jitter on the player enjoyment.

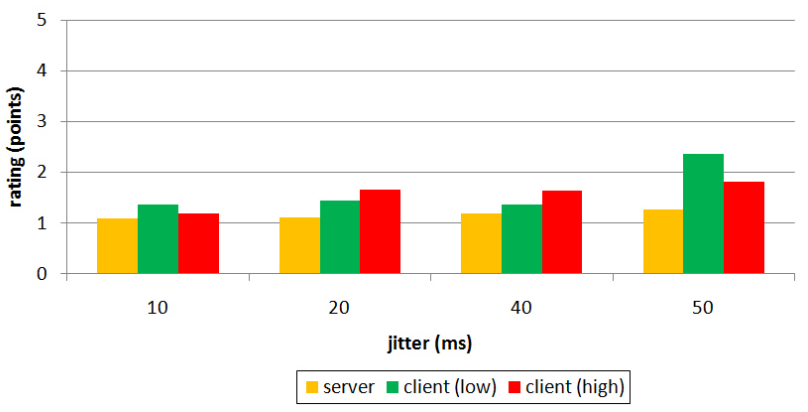

(c) Influence of jitter on the player frustration.

Figure 12: Comparison of responses given by impaired and unimpaired players.

shared activities, etc. Based on the analysis provided in section 4.1 we conclude that delays up to $100 \mathrm{~ms}$ can be considered as acceptable for the game being used. Users perceive their experience as degrading when one-way delay values exceed $100 \mathrm{~ms}$.

Our second study focuses on the impact of jitter on cooperation in games. We have found that jitter has a negative influence on user performance, in particular on the task completion time. At the same time players have not perceived this influence as a degradation of their experience. The correlation between the completion time and the jitter level (obtained from the objective measures) was not reflected in the subjective evaluation. Most given ratings indicated a very low negative impact on cooperative play almost equal among players with different conditions. Nevertheless, when the goal of the game became time dependent, we have observed a difference between ratings given by players who were not directly affected by the network jitter and players who experienced low or high jitter. We also found a growing negative influence of jitter on players who were affected directly. The differences between affected and non-affected players have confirmed our expectation of the nega- tive influence of jitter on the gaming experience. Jitter higher than $50 \mathrm{~ms}$ introduces a great discrepancy between responses given by impaired and unimpaired players.

With our study we made the first attempt to evaluate the influence of network quality on the cooperative games, which we based on Little Big Planet 2. Although we realize that the results obtained in our study may be game-dependent, we believe that they are applicable to other games involving similar types of interaction between players. Of course further analysis is absolutely necessary. Other aspects of cooperation as well as other cooperative games need to be analyzed.

\section{References}

Armitage, G., And Stewart, L. 2004. Limitations of using real-world, public servers to estimate jitter tolerance of first person shooter games. In Proceedings of the 2004 ACM SIGCHI International Conference on Advances in computer entertainment technology, ACM, 257-262.

Chen, K.-T., Huang, P., And LeI, C.-L. 2006. How sensitive are online gamers to network quality? Commun. ACM 49 (November), 34-38.

Dick, M., Wellnitz, O., AND Wolf, L. 2005. Analysis of factors affecting players' performance and perception in multiplayer games. In Proceedings of 4th ACM SIGCOMM workshop on Network and system support for games, ACM, 1-7.

Garapati, N., 2009. Delay impact on gaming experience for a first person shooter game, available at http://www.bth.se/.

HEMminger, S. 2005. Network emulation with netem.

Jehaes, T., De Vleeschauwer, D., Coppens, T., VAN DoORselaer, B., Deckers, E., NAudts, W., SPRUYT, K., AND SMETS, R. 2003. Access network delay in networked games. In Proceedings of the 2nd workshop on Network and system support for games, ACM, 63-71.

KIM, J., PARK, I., AND SHIM, K. 2007. The effects of network loads and latency in multiplayer online games. In Entertainment Computing ICEC 2007, vol. 4740 of Lecture Notes in Computer Science. Springer Berlin / Heidelberg, 427-432.

PAntel, L., AND Wolf, L. C. 2002. On the impact of delay on real-time multiplayer games. In Proceedings of the 12th international workshop on Network and operating systems support for digital audio and video, ACM, 23-29.

PARK, K. S., AND KenYon, R. V. 1999. Effects of network characteristics on human performance in a collaborative virtual environment. In Proceedings of the IEEE Virtual Reality, IEEE Computer Society, Washington, DC, USA, 104-111.

QuAX, P., MONSIEURS, P., LAMOTTE, W. De Vleeschauwer, D., And Degrande, N. 2004. Objective and subjective evaluation of the influence of small amounts of delay and jitter on a recent first person shooter game. In Proceedings of 3 rd ACM SIGCOMM workshop on Network and system support for games, ACM, 152-156.

Steed, A., AND Oliveira, M. 2009. Networked Graphics: Building Networked Games and Virtual Environments. Morgan Kaufmann. Elsevier Science.

Stuckel, D., AND Gutwin, C. 2008. The effects of local lag on tightly-coupled interaction in distributed groupware. In Proceedings of the 2008 ACM conference on Computer supported cooperative work, ACM, 447-456. 